\title{
Intraoperative Ocular Injury, CTCAE
}

National Cancer Institute

\section{Source}

National Cancer Institute. Intraoperative Ocular Injury, CT CAE. NCI Thesaurus. Code C143608.

A finding of damage to the eye during a surgical procedure. 